\title{
WEAR ANALYSIS OF CAM AND FOLLOWER USING FINITE ELEMENT METHOD
}

\author{
NEGA TESFIE ASFAW \\ Department of Mechanical Engineering, Institute of Technology - Hawassa University, Ethiopia
}

\begin{abstract}
A particular type of contact condition, know as cam and follower contact, exists in the direct valve train system of an engine and is partly responsible for wear. The wear analysis of cam and follower contact system are analyzed with respect to the cam angle and the pressure angle and finite element modeling of cam and follower assembly is done using the ABAQUS analysis software. The contact pressure, von misses' stresses are calculated theoretically and the ABAQUS results are presented in contour plot and numerically. The results showed that the cam rotational angle and the pressure angle had an effect on the contact pressure, on stress distribution and also plays a great role on the surface wear of the contact. The results also showed that the contact pressure, von misses stress and the wear increases with of cam rotational angle and the wear increases linearly with the contact pressure. Based upon the theoretical analyses and ABAQUS analysis, a theoretical model for evaluating the tribological performance of the valve train was developed. A multi-aspect comparison between theoretical and ABAQUS results was made. A good agreement between theoretical and ABAQUS analysis results showed that the model provided a reliable prediction of the tribological characteristics of the cam/roller follower. Three critical portions of the cam could be identified these are the cam basic surface region, cam flank and cam lobe region. The most critical region is the cam lobe region.

KEYWORDS: Cam, Follower, Grey Cast Iron, Surface Wear, Finite Element Method, Abaqus
\end{abstract}

Received: Jun 06, 2020; Accepted: Jun 26, 2020; Published: Aug 24, 2020; Paper Id.: IJMPERDJUN2020866

\section{INTRODUCTION}

Cams are commonly used in opening and closing of valves in internal combustion engines. Both the inlet and outlet valves are regulated using cam and follower. The study of cam and follower mechanism becomes important for desired and required performance of the engines [1]. A cam-follower kinematic pair works under complicated conditions of mechanical load, and wears during operation. The contact surfaces of the cam and the follower are usually surface hardened. The hardening may be due to phase transformation or precipitation processes occurring in the material during heat treatment or thermo-chemical treatment [4].

Wear can be defined as the removal of material from solid surfaces as a result of mechanical action. The amount of material removed is often quite small for contact surfaces in high performance machines or machines with long life. Wear can appear in many ways depending on the material of the interacting contact surfaces, the environment and the running conditions. From an engineering point of view, wear is often classified as mild or severe. Mild wearis what the engineers strive for. That can be obtained by proper form and topography of the contact surfaces. [5]

Scuffing, Pitting polish, and scoring are the kinds of modes cam and follower wear occurs from the surface due to the high contact stresses that are present between cam and follower contact. Pitting most often occurs in a follower type timing gear system with the camshaft inside the engine block. It is a failure of surface manifested by 
break out of small roughly triangular portions of a material surface due to compressive stresses causing fatigue at a point below a surface. Scuffing is a local welding of two heavenly loaded surfaces specially when a high degree of relative sliding occurs under poor lubrication conditions, followed by tearing apart of the welded material. It starts at high stressed zone with poor surface finish and generally during the running-in period of new parts. Polishing wear is because of the general attrition of contacting surfaces. scoring is scratches in the surface. Most methods of scuffing investigation concern an engine with overhead camshafts. The thickness of the oil film is too small to ideally separate all the irregularities of the mating surfaces it may lead to welding and breaking of the connections between the peaks of the irregularities in the contact micro areas [6].

\section{MATERIALS AND METHODS}

The preferred material for a cam mating with a slide cam follower at indirect valve drive is grey cast iron w10,11x. It has the following composition: $3.1-3.5 \% \mathrm{C}, 1.8-2.5 \% \mathrm{Si}$, max. $0.15 \% \mathrm{~S}$, max. $0.2 \% \mathrm{P}, 0.5-0.8 \% \mathrm{Mn}, 0.15-0.25 \% \mathrm{Ni}, 0.7-1 \%$ $\mathrm{Cu}, 0.15-0.25 \% \mathrm{MO}$, the rest is $\mathrm{Fe}$. The cam surface gets hardened during primary crystallization. The minimum hardness should be $45 \mathrm{HRC}$ with a 3-mm hardened layer at the lobe and the flanks.

The mating follower should be made of chilled cast iron, hardened and tempered, with an increased content of $\mathrm{Ni}, \mathrm{Cr}, \mathrm{Mo}$, V. It has the following composition 3-3.6\% C, 2-2.8\% Si, max. 0.12\% S, max. 0.25\% P, 0.4-1\% Mn, 0.4 $0.7 \% \mathrm{Ni}, 0.9-1.25 \% \mathrm{Cr}, 0.4-0.7 \% \mathrm{Mo}$, the rest was Fe. The minimum hardness of the follower should be $54 \mathrm{HRC}$ with the depth of the hardened layer being $3 \mathrm{~mm}$.

\section{ANALYTICAL METHOD IN WEAR ANALYSIS OF CAM AND FOLLOWER}

\section{Wear Analysis}

When two bodies are in contact and are in relative motion with respect to each other, wear is expected to develop on the regions of contact. The type of contact that the bodies experience is dependent on how the bodies move relative to each other. One type of contact condition that is of interest is the oscillatory contact. This type of contact condition is characterized by an oscillatory relative motion between the bodies that are in contact. The contact between a cam and follower is an example of this type contact.

Generally, wear does not involve a single mechanism, therefore it is advisable to take an integrated wear analysis approach assuming the wear behavior as a system property. In other words, wear analysis is not limited to the evaluation of the effects of materials on wear behavior, but recommends changes in contact geometry, roughness, tolerance, and so on so that overall favorable results can be achieved. it is probably accurate to say that there is little incentive for a designer to use any of the wear-equations available in the literature. A scan of many wear models shows considerable incongruity. Equation have either too many undefined variables or too few variables to adequately describe the system". Most of available equations are derived/made for mild wear rate of components. Therefore; it can be said that to estimate wear theoretical equations, experimental coefficients are required.

Most wear models assume linearity, and they often also assume that the wear is directly proportional to the local contact pressure. The most common wear model is named Archard's Wear Law, although Holm formulated the same model much earlier than Archard. However, Archard and Holm interpreted the model differently. 
So the above listed wear mechanisms can be described by archad's wear equation. The model is expressed mathematically as follows;

Then the wear volume can be expressed as follows:

$$
V=\frac{K *(F g-F i n) * S}{H \cos \phi}
$$

There is small sliding in case of cam and follower contact. So, the wear volume can be expressed as the rate of sliding distance. The wear volume per sliding can be expressed as follows;

$$
\mathrm{Q}=\frac{K * F_{n}}{H}
$$

$\mathrm{V}$ is the wear volume, $\mathrm{Q}$ is the wear volume per unit sliding,$F_{n}$ is normal load, $\mathrm{H}$ is the hardness of the material

Where $V$ is the volume lost, $s$ the sliding distance, $K$ the dimensionless wear coefficient, $H$ the Brinell hardness of the softer material, and $F_{n}$ the normal force. The non-dimensionedwear coefficient $K$ and the hardness are bundled up into a single dimensioned wear coefficient $k(\mathrm{~Pa}-1)$. It should be noted that the wear coefficient $k$ is not an intrinsic material property but is also dependent on the operating condition. The value of $k$ for a specific operating condition and given pair of materials may be obtained by experiments. Also, worth noting, is that measured values of wear coefficients usually have large scatter and may affect wear predictions significantly. Care should thus be taken in obtaining these values $\mathrm{K}$ is the wear coefficient which distinguish between the wear mechanisms by considering the area subjected to wear it is possible to express the wear displacement in terms of the contact pressure.

Dividing both sides by the subjected area

$$
\frac{Q}{A}=\frac{K \times F_{n}}{H A}
$$

Where $\mathrm{K}$ is a dimensionless quantity which is usually called the wear coefficient and which provides a valuable means of comparing the severity of different wear processes.

For engineering applications, and especially for the wear of materials whose hardness cannot readily be defined the wear rate is commonly stated as:

$$
\frac{K}{H}=k_{i}=\frac{W}{F_{n}}
$$

$k_{\mathrm{i}}$ is often called the specific wear rate and quoted in units of $\mathrm{mm}^{3} \mathrm{~N}^{-1} \mathrm{~m}^{-1}$. For a material with a hardness $\mathrm{H}$ of 1 GPa (a soft steel, or a hard aluminum alloy, for example), the numerical value of $\mathrm{k}$ expressed in $\mathrm{mm}^{3} \mathrm{~N}^{-1} \mathrm{~m}^{-1}$ is exactly the same as the value of $\mathrm{K}$.

\section{Abrasive Wear}

If the wear rate is given by a specific wear rate, $\left(w_{s}=\right.$ wear volume/load $\times$ sliding distance $)$, or a wear coefficient, $\left(\mathrm{K}=w_{s}\right.$ $\times \mathrm{H}$, where $\mathrm{H}$ is the hardness), they are derived from equation as follows:

The wear can also be expressed as a function of sliding velocity

$$
V=k \times p \times v_{s} \times t
$$

Where $\mathrm{p}$ is the contact pressure 
$v_{\mathrm{s}}$ is the sliding velocity

This sliding velocity is the part of tangential velocity at the contact point

$\mathrm{vt}=\mathrm{X} \mathrm{x}^{*} \theta \mathrm{cam}$

Where $\mathrm{X}$ is the distance from the center of cam to the contact point that is the position of follower along the cam profile

The wear can be expressed as

For the rising action,

$V=k \times p \times\left(\mathrm{rb} \times 0.5 \mathrm{~h}\left(1-\cos \pi \frac{\theta}{\theta_{\mathrm{A}}}\right)\right) \theta_{\mathrm{cam}} \times \cos \alpha \times t$

For the returning action,

$V=k \times p \times\left(\mathrm{rb} \times 0.5 \mathrm{~h}\left(1-\cos \pi \frac{\theta-\theta_{c}}{\theta_{b}-\theta_{c}}\right)\right) \theta_{\operatorname{cam}} \cos \alpha \times t$

Under the cam lobe there is two cases

- The rising action

- The returning action

For the rise action the roller is in contact with the cam from $147^{\circ}$ to 180 . The pressure, the distance from the center of cam to the contact point, and the cosine value of the pressure angle are maximum when the pressure angle is 180.this results the wear is sever at the top of the cam lobe. The wear value can be expressed as

$V=k \times p_{\max } \times x_{\max } \theta_{\operatorname{cam}} \cos \phi \times t$

$V=k \times p_{\max } \times \mathrm{x}_{\max } \theta_{\mathrm{cam}} \times \cos \phi \times t_{\text {is }}$ the maximum wear at the top of the cam lobe. So, for the lifting action the wear decreases with the increase of cam angle. For the lowering action the roller moves from 180 to $212^{0}$. The maximum wear attains at the top i.e. at $180 \mathrm{deg}$. for this case the wear increases with the increase of the cam angle.

So, the maximum wear is generally attains at the top the cam i.e. at the cam lobe that is at the $180^{\circ}$.

The instantaneous rate of wear $\mathrm{i}(\mathrm{x}, \mathrm{t})$

$$
i(x, t)=k \times p \times h \times G \times V s
$$

For $\mathrm{G}$ in this equation is a semi elliptical pressure distribution is assumed in theoretical approach. Then the wear occurring during a single passage of contact cam surface is given by

$$
\mathrm{W}=\int_{\mathrm{t} 1}^{\mathrm{t2}} k \times p \times h \times G \times \mathrm{Vs} \mathrm{dt}
$$

Where $t_{1}$ and $t_{2}$ are the time at the start and end of contact point on cam surface.

\section{Basic Circle Surface Wear}

The rate of wear is $Q=\frac{K \times \text { Fn } \times \text { w w }}{H}$ 
Here the wear coefficient, the radius of basic circle, the hardness are constants throughout the analysis, and for the analysis the angular speed is taken to be 1200rpm. Then the wear rate is directly proportional to the normal force applied on the cam basic circle surface. The pressure angle is constant throughout this surface.

Where $\mathrm{r}$ is basic circle radius, $\Theta$ is cam rotation angle (angular position of cam)

At the basic circle surfaces the pressure angle is $0^{\circ}$. The effect is constant throughout the basic circle surfaces and spring force is small and the contact area is high this shows low pressure is produced this result low wear rate(volume). So, the wear rate is not severed and negligible plastic deformation due low pressure produced and temperature is produced on this surface.

\section{Cam Flank Wear}

The change in the shape of the surface during wear leads to a redistribution of thecontact stresses. The shape of cam flank is flat surface. That means it is exposed for sliding and rolling action. Here it exposed for more sliding action. This sliding causes different types of wear mechanisms i.e. pitting, scuffing, and scoring. Archard's law stays at the basis of different wear mechanisms and the following relation between the rate of wear, the contact pressure and the rate of slippage was proposed:

$$
w=\int_{\Omega 1}^{s 2} k \times p \times \partial s
$$

Here the sliding distance can be measured directly. The flank length can be divided into different parts. Each part is exposed to different wear amount. The flank part covers from $82^{\circ}$ to $147^{\circ}$ of the cam rotation angle.

\section{Wear Displacement}

The wear displacement can be derived from the wear volume. So, the rate of wear displacement with respect to the sliding distance is directly proportional to the contact pressure and inversely proportional to the hardness of the material. the sliding distance cam be expressed as a function of time and sliding velocity this depicted below

Let us consider rollers follower and cam of radius R1 and R2 respectively. The follower and cam are pressed together by force $F_{N}$ and rotate at angular velocities $\omega_{1}$ and $\omega_{2}$, respectively. The peripheral velocities of the contact surfaces are $v_{1}=\omega_{1} \cdot R_{l}$ and $v_{2}=\omega_{2} \cdot R_{2}$. The wear of the contact surfaces is assumed to be properly described by the following wear model:

$$
\frac{d h_{i}}{d t}=k_{i} \times p \times V_{i_{i} g}
$$

Where $i=1$ for cam and $i=2$ for roller follower; $h_{i}$ is the wear depth at a point on surface $I$ when it rubs against the opposite contact surface, $k_{i}$ is the wear coefficient for a point onsurface $i$ when it rubs against the opposite contact surface, $p$ is the local contact pressure, and $v_{s}, i$ is the sliding velocity at a point on surface $i$ sliding against the opposite interactingsurface. The sliding velocity, $v_{s}, i$, for points on both contact surfaces equals.

This integral equation thus can be reformulated as follows

$$
h_{\text {inew }}=h_{\text {iold }}+\int_{t_{1}}^{t_{2}}\left(k_{i} \times \mathrm{p} \times\left|V_{1}-V_{2}\right|\right) d t
$$

From this equation $k_{\mathrm{i}}$, and $\mathrm{R}$ are constants and 
$\omega * d t$ is the infinitesimal angular position of the ratating cam

$h_{\text {inew }}=h_{\text {iold }}+k_{i} \times R \int_{\theta_{1}}^{\theta_{2}} P d \theta$

This can describe the wear displacement can be estimated in terms of the cam angle.

\section{MODELING OF 3D CAM AND FOLLOWER}

In this section finite element approach is used for contact stress analysis in cam and follower system. The approach is based on application of ABAQUS software version 6.10CAE computer programs.

\section{Modeling the 3D Cam and Follower}

To model the system the geometry cam and follower must be known that is the basic circle radius of cam, radius of cam lobe, radius of follower and the maximum lift must be specified. These parameters are depicted below:

Table 1: The Geometrical Values of Cam and Follower

\begin{tabular}{|l|c|}
\hline \multicolumn{1}{|c|}{ Elements } & Dimensions \\
\hline Cam Basic Circle Radius & $24 \mathrm{~mm}$ \\
\hline Cam Lobe Radius & $13 \mathrm{~mm}$ \\
\hline Roller Radius & $9 \mathrm{~mm}$ \\
\hline
\end{tabular}

The model can be generated using the above values

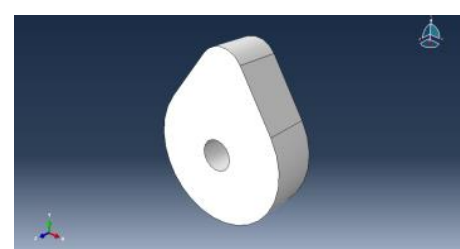

Figure 1: 3D Cam Model

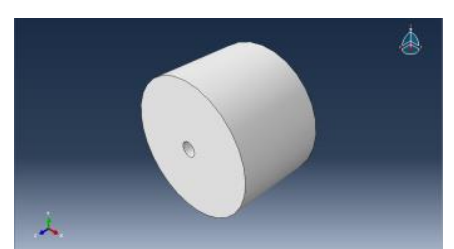

Figure 2: 3D Roller Model

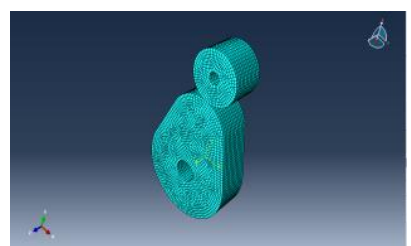

Figure 3: Meshed Assembly

\section{The Materials Spécification}

Cam and follower are made from the same material of different hardness. The material used is cast iron so the material properties of cast iron must be specified i.e. the modulus of elasticity, poisons ratio, density.

Table 2: Material Properties of Cast Iron

\begin{tabular}{|l|c|c|}
\hline \multicolumn{1}{|c|}{ Material Property } & Value & Units \\
\hline Modulus of Elasticity & 83 & $\mathrm{Gpa}$ \\
\hline Poisons Ratio & 0.29 & unitless \\
\hline Density & 7200 & $\mathrm{Kg} / \mathrm{m}^{3}$ \\
\hline
\end{tabular}

Abaqus divided these properties as general property and elastic property i.e. density is the general property of material and the modulus of elasticity and poisons ratio are the elastic properties of material.

\section{RESULT AND DISCUSSION}

\section{Contact Pressure}

The comparison of contact pressure between the theoretical and ABAQUS results is done and results are summarized in table below. 
Table 3: ABAQUS and Theoretical Results Numerically

\begin{tabular}{|l|c|c|}
\hline \multicolumn{1}{|c|}{ Region } & Max. Theoretical Result & Max. Abaqus Result \\
\hline Cam Basic Surface & $701.825 .1 \mathrm{Mpa}$ & $552.856 \mathrm{Mpa}$ \\
\hline Cam Flank & $716.975 \mathrm{Mpa}$ & $844.985 \mathrm{Mpa}$ \\
\hline Cam Lobe & $1025.15 \mathrm{Mpa}$ & $1441.31 \mathrm{Mpa}$ \\
\hline
\end{tabular}

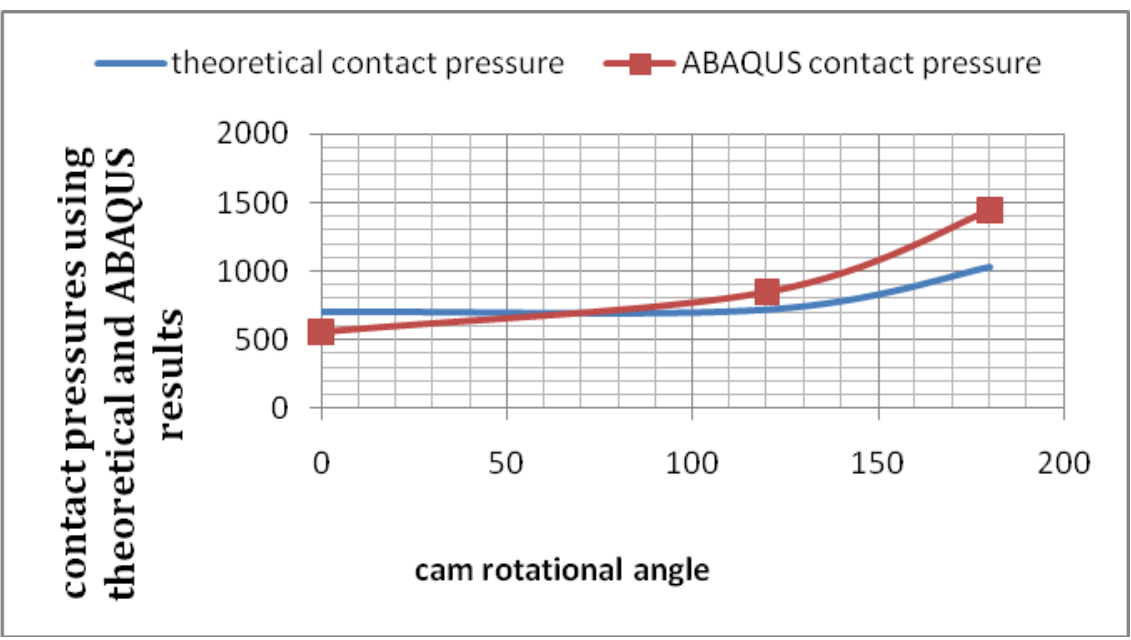

Figure 4: Contact Pressure Distribution.

\section{Vonmisses Stresses}

The comparison of von misses between the theoretical and ABAQUS results is done and results are summarized in table below.

Table 4: Comparison of Von Misses between the Theoretical and ABAQUS Results

\begin{tabular}{|l|c|c|}
\hline \multicolumn{1}{|c|}{ Region } & Theoretical Results & ABAQUS Results \\
\hline Basic Circle Region & $356.2 \mathrm{Mpa}$ & $387.759 \mathrm{Mpa}$ \\
\hline Cam Flank & $363.2 \mathrm{Mpa}$ & $527.79 \mathrm{Mpa}$ \\
\hline Cam Lobe & $520.1 \mathrm{Mpa}$ & $773.95 \mathrm{Mpa}$ \\
\hline
\end{tabular}

This can be summarized using the fig. as follows:

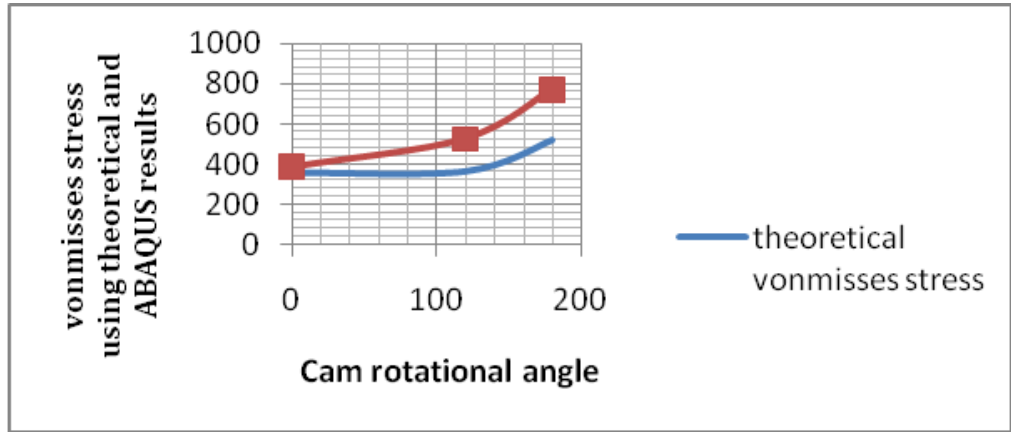

Figure 5: Von Misses Stress Distribution.

\section{Cam and Follower Wear}

The wear of cam and follower can be also calculated using the results of ABAQUS analysis software. The wear rate can be summarized using graph. 


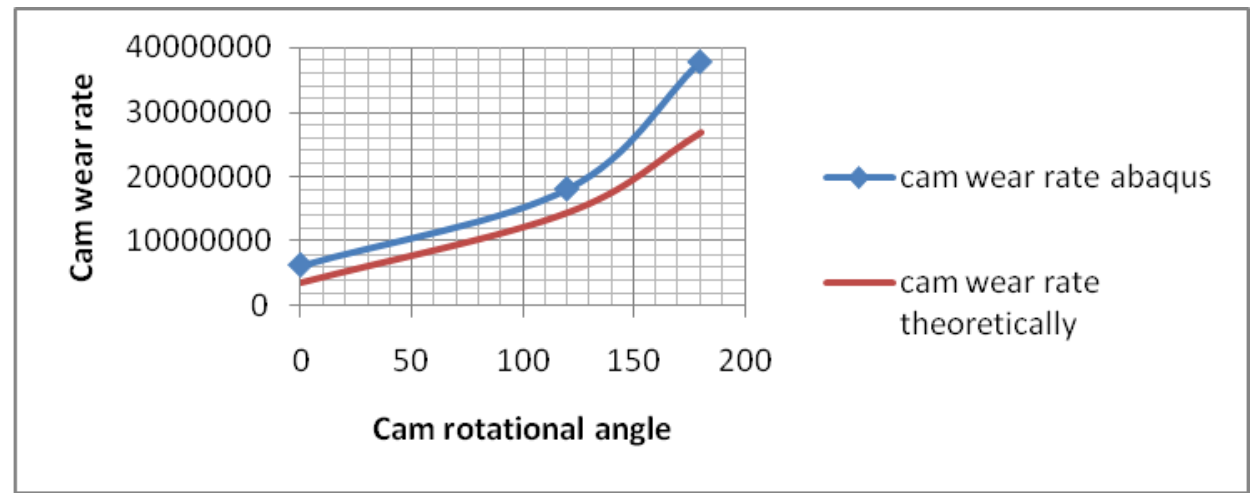

Figure 6: Cam Wears Distribution.

If we analyze the comparison of wear calculated from the contact result of ABAQUS software and theoretical contact pressure results, both theoretical and ABAQUS results of wear is in good agreement the wear increases with the increase of cam rotation angle, and the wear is also increases linearly with the contact pressure.

The distribution of follower wear is depicted below it shows that in the roller follower wear rate increases linearly with the cam wear rate. The cam rotation angle had an effect on follower wear rate i.e. the roller follower wear rate increases with the increase of cam angle.

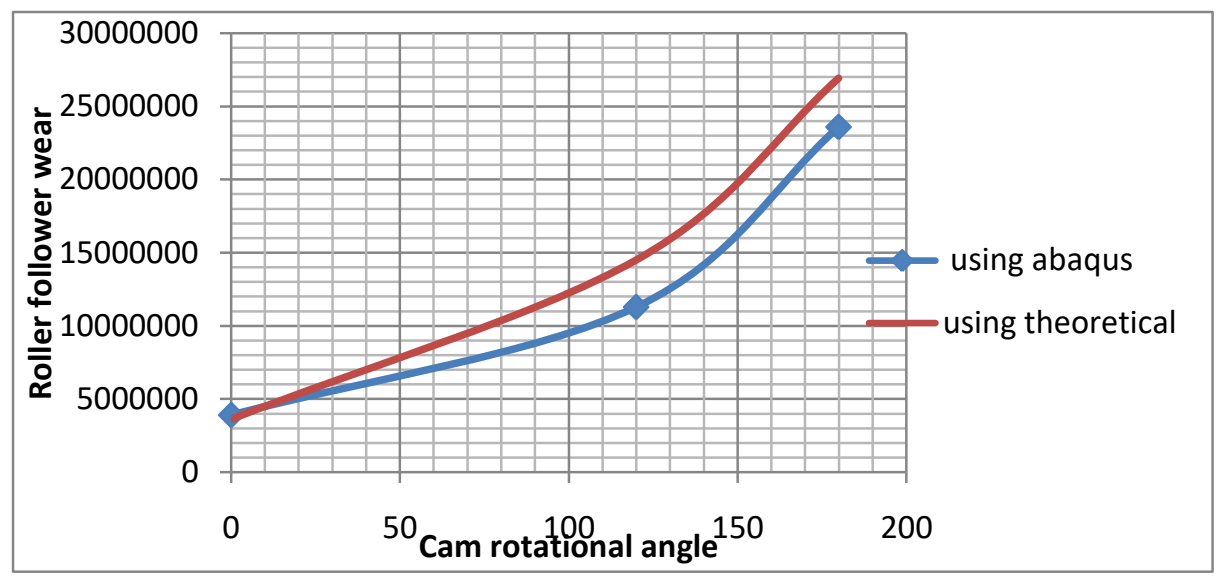

Figure 7: Roller Follower Wears Distribution.

\section{CONCLUSIONS}

- A multi-aspect comparison between the theoretical predictions and the ABAQUS results has been undertaken. The main conclusions which can be obtained from these theoretical and ABAQUS results and theoretical comparisons are summarized below;

- The good agreement between the theoretical predictions and ABAQUS results in many aspects shows that the theoretical model developed in this chapter provides a good basis for the prediction of tribological characteristics of the cam/roller follower and associated Mechanism.

- Both ABAQUS observation and the theoretical analysis predict that the cam rotational angle has a significant influence not only on the actual load on the cam but also on the surface wear of the contacting element. Whilst the pressure angle has a major influence on the follower motion even creating jam unless it is specified so the effects of the follower/guide contact can also be important and must be taken into consideration. 
- Both the theoretical and ABAQUS software results show that the contact pressure increases with the increase of cam angle for the rising action of the valve train. by comparing the von misses predicted by the theoretical analysis is in good agreement with the ABAQUS analysis results in the case of increasing the von misses stress with respect to the cam angle

\section{ACKNOWLEDGEMENTS}

I thank the management of Institute of Technology - Hawassa University and Institute of Technology- Addis Abbaba University, Ethiopia.

\section{REFERENCES}

1. De Abreu Duque, P., de Souza, M., Savoy, J., and Valentina, G., 2011, Analysis of the Contact Pressure between Cams and Roller Followers in Assembled Camshafts, SAE Technical Paper, 36, 247 - 256.

2. J. Michalski, J. Marszalek, K. Kubiak, 2000, An experimental study of diesel engine cam and follower wear with particular reference to the properties of the materials, Wear, 35, $959-966$.

3. Ramesh, B., S. MOHAMMED IQBAL, and K. LOGESH. "Aero-Structural Optimization of Aircraft Wing by Finite Element Method." International Journal of Mechanical and Production Engineering Research and Development (IJMPERD) 7.6 (2013): 409-416.

4. Muhammad Khurram, Riaz Ahmad Mufti \&Muhammad Umar, 2019, Simulating cam and follower wear in valve train, Journal Tribology - Materials, Surfaces \& Interfaces, 14, 59 - 65.

5. Saravanan, A., et al. "Static analysis and weight reduction of aluminum casting alloy connecting rod using finite element method." Int J Mech Prod Eng Res Dev (IJMPERD) 8.3 (2018).

6. Per Lindholm, Stefan Björklund, Miguel Calvo Cortes, 2003, Characterization of wear on a cam follower system in a diesel engine, Wear, 254, $1199-1207$.

7. M Kushwaha and H Rahnejat, 2002, Transient elastohydrodynamic lubrication of finite line conjunction of cam to follower concentrated contact, Journal of Physics D: Applied Physics, 35, 546 - 554.

8. Saravanan, A., et al. "Design and analysis of trestle hydraulic jack using finite element method." International Journal of Mechanical and production Engineering Research and Development 8 (2018): 437-448.

9. NagarajNayak. P, A. Lakshminarayanan, M.K. GajendraBabu, A.D.Dani, 2006, Predictions of cam follower wear in diesel engines, Wear, 260, $181-192$.

10. Georgantzinos, Stelios K. "The Role of Multi-Scale Finite Element Methods in Analysis and Design of Advanced Mechanical and Aerospace Structures." Nanotechnology 10.15 (2019): 10-12. 

